\title{
Frequency Characteristics of the Complex Permeability of Ferrites
}

\section{H.SAOTOME and K.TACHIBANA}

\author{
Department of Electrical \& Electronic Engineering, Chiba University \\ 1-33 Yayoi, Inage, Chiba 263, Japan
}

\begin{abstract}
Exciting ferrite cores in a high frequency range, the real part of the complex permeability deteriorates and the imaginary part of it has a peak after starting the deterioration. For a certain $\mathrm{Mn}-\mathrm{Zn}$ ferrite material, we find that the frequency characteristics of the complex permeability depend on core size, i.e. the real part decreases more sharply with an increase in frequency when core size becomes larger. In order to demonstrate this phenomenon, an electric circuit with a capacitor is assumed for a ferrite core. It is examined whether its capacitance correlates with the magnetic loss or the eddy current loss. As a result, it is found that the core size dependence of the frequency characteristics is caused by the dielectric currents flowing in the cores.
\end{abstract}

\section{INTRODUCTION}

The complex permeability $\mu^{\prime}-j \mu^{\prime \prime}$ should express only magnetic and magnetizing characteristics of a material. However, it is often difficult to remove conductive and dielectric characteristics of the material in measurements. For two Ni-Zn ferrites of which the conductivity and the permittivity are small in view of loss separate analysis, it is found that the frequency characteristics of their complex permeability can be derived from an inductance-resistance-parallel circuit where the resistance consume the magnetic loss power $[1,2]$. This parallel circuit is shown by $L$ and $R_{M}$ in Fig. 1. On the other hand, some $\mathrm{Mn}-\mathrm{Zn}$ ferrites need additional circuit elements which are connected in parallel to the $L-R_{M}$ circuit, such as a resistance $\left(R_{E}\right)$ and a capacitance-resistance-series element $\left(C-R_{D}\right)$ for consuming the eddy current and dielectric loss power, respectively (Fig. 1) [3,4].

By taking these circuit elements into account, the frequency characteristics of the complex permeability for a $\mathrm{Mn}-\mathrm{Zn}$ ferrite core is calculated and compared with the measured ones as shown in Fig. 2(a) where they are plotted as the relative permeability. As a result, good agreements are obtained between them. The core size is listed in Table $I$ as a small core and the exciting condition is $B_{m}=20 \mathrm{mT}$ which is the peak value of the sinusoidal flux density of the core. In the calculation, the skin effect is taken into account, i.e. we have solved the governing equation for the magnetic field, derived from the Maxwell's equations, with

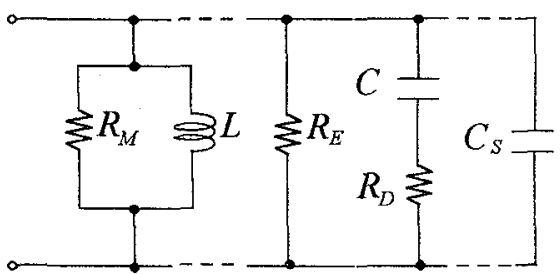

Fig. 1. Equivalent circuit of a ferrite core. the Bessel functions [2-4]. Therefore, $B_{m}=20 \mathrm{mT}$ exactly means the average value over the crosssectional area of the magnetic flux path.

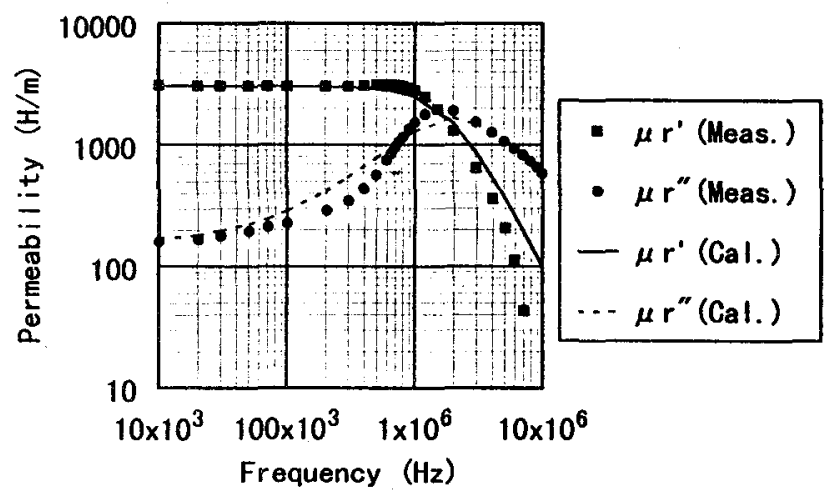

(a) Small core.

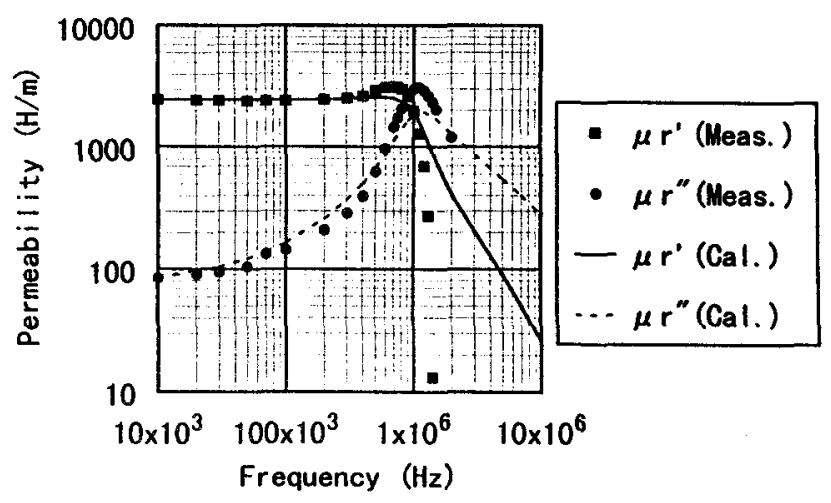

(b) Large core.

Fig. 2. Permeability without taking account of $C_{S}$.

Table I Core Dimensions

\begin{tabular}{llll}
\hline & $l(\mathrm{~mm})$ & $A\left(\mathrm{~mm}^{2}\right)$ & $l / A\left(\mathrm{~mm}^{-1}\right)$ \\
\hline Small core & 28.4 & 9.00 & 3.16 \\
Large core & 196 & 104 & 1.89
\end{tabular}

$l$ and $A$ are the length and the cross-sectional area of a magnetic flux path, respectively. 
In contrast with the previous results, in the case of the large core whose dimensions are listed in Table I, the computed frequency characteristics do not coincide with the measured ones in the high frequency region (Fig. 2(b), $B_{m}=20 \mathrm{mT}$ ), even though the skin effect of the dielectric current (flowing the $C-R_{D}$ element) which is larger than that of the small core has been taken into account. By comparing the experimental data in Figs. 2(a) and 2(b), it is found that the complex permeability depends on core size. In order to explain this phenomenon, an additional dielectric element having no loss is assumed, where its permittivity is the same for the small and large cores. This element is shown by $C_{S}$ as a lumped circuit in Fig. 1. This paper shows whether the additional dielectric element correlates with the magnetic loss or the eddy current loss of the ferrite from the core-size-dependent point of view.

\section{II . STRAY CAPACITANCE OF WINDINGS ?}

We studied effect of stray capacity of windings in measurement of the complex permeability, before estimating value of $C_{S}$ of each sample. Three different structures of winding for the large core are shown in Fig.3. The measured complex permeabilities for the cores of Figs.3(a), 3(b) and 3(c) are plotted in Fig.4, where $B_{m}=20 \mathrm{mT}$. The values of the stray capacitance for the windings are different. However, it is found the difference has negligible influence on measuring the complex permeability for the core.

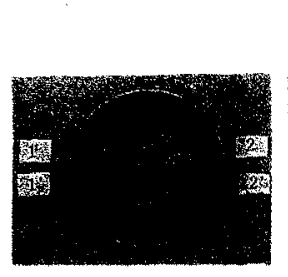

(a)

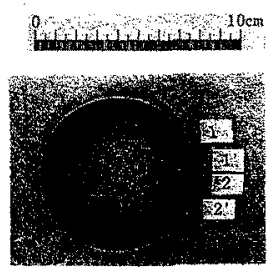

(b)

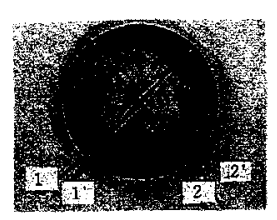

(c)
Fig. 3. Three different types of windings.

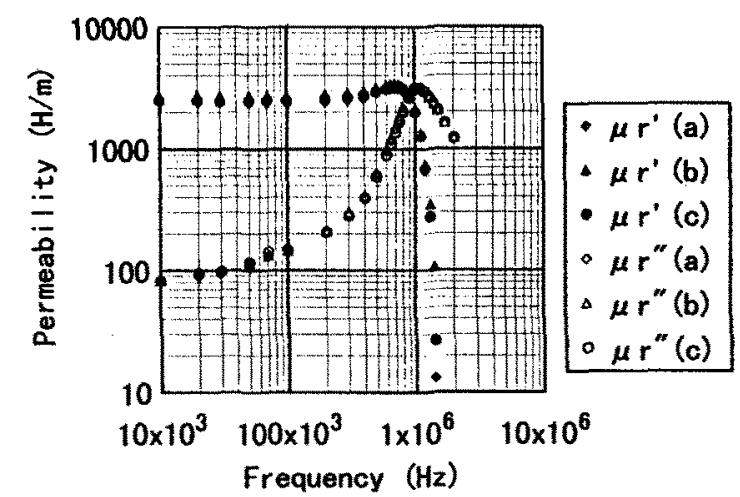

Fig. 4. Measured complex permeabilities with the windings.

\section{CORRELATION WTTH THE MAGNETTC AND ELECTRIC PROPERTY}

For a toroidal core with a circular cross-section of a magnetic flux path, $R_{M}$ and $R_{E}$ are respectively given by

$$
\begin{aligned}
& R_{M}=\lambda A N^{2} / l \\
& R_{E}=8 \pi N^{2} /(\sigma l)
\end{aligned}
$$

where $N, \lambda$ and $\sigma$ are the number of turns, the magnetic loss parameter and the DC conductivity of the core, respectively [2]. Equations (1) and (2) are valid for low frequency excitation. From (1) and (2), it is found that $R_{M}$ is proportional to the cross-sectional area of a magnetic flux path, $A$, whereas $R_{E}$ is independent of $A$. In this chapter, we assume and

$$
C_{S}=\varepsilon_{S} l /\left(A N^{2}\right) \propto 1 / R_{M} \text { (geometrically) }
$$

$$
C_{S}=\varepsilon_{S} l /\left(8 \pi N^{2}\right) \propto l / R_{E} \text { (geometrically) }
$$

with a constant permittivity $\varepsilon_{S}$. If (3) is correct, $C_{S}$ is supposed to be caused by magnetic resonance because its reciprocal is proportional to $R_{M}$ and the inductance $L$ with respect to the core dimensions. On the other hand, if (4) is substantiated, the dielectric current flowing in parallel to the eddy current should exists. The geometrical direction of the dielectric current is the same as that of the eddy current and the electric field, however, its phase angle related to the electric field is 90 degree lead. This result implies that three different phase currents flow in the core, i.e. voltage-in-phase, lead and completely lead currents flow in $R_{E}, C-R_{D}$ and $C_{S}$, respectively.

The problem discussed above should be analyzed with consideration of the skin effect of the fields. Therefore, we obtained the magnetic and electric field distributions with the Bessel functions and calculated the impedance of the cores. The analysis is easily carried out by applying the complex medium parameters $\dot{\lambda}$ and $\dot{\sigma}$ to the solutions of the fields. We assumed $1 / \dot{\lambda}=\delta^{\prime}+j \delta^{\prime \prime}$ and $\dot{\sigma}=\sigma^{\prime}+j \sigma^{\prime \prime}$ where $\delta^{\prime \prime}=\omega \varepsilon_{S}$ and $\sigma^{\prime \prime}=\omega \varepsilon_{S}$, respectively, with the angular exciting frequency $\omega$.

Assuming $\delta^{\prime \prime}=2.74 \times 10^{-12} \omega$ and ignoring $\sigma^{\prime \prime}$, the frequency characteristics of the complex permeability for the large core are calculated and plotted in Fig.5(a) with the measured data. We fitted $\varepsilon_{S}$ in order to make the computational values coincide with the experimental data. With the same condition, the complex permeability for the small core is calculated and plotted in Fig.5(b) with its measured data. In Fig.5(b), the computational and measured data do not coincide. This means we find out no consistent and constant $\varepsilon_{S}$, in core dimensions, corresponding to that of (3). 


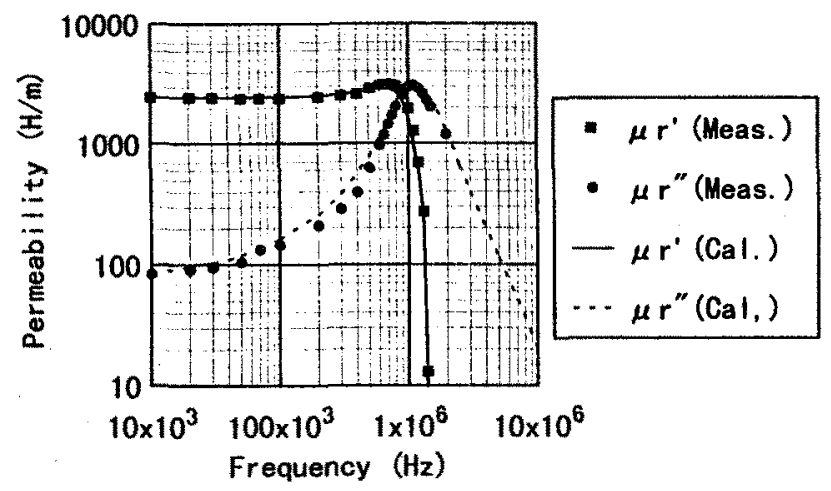

(a) Large core.

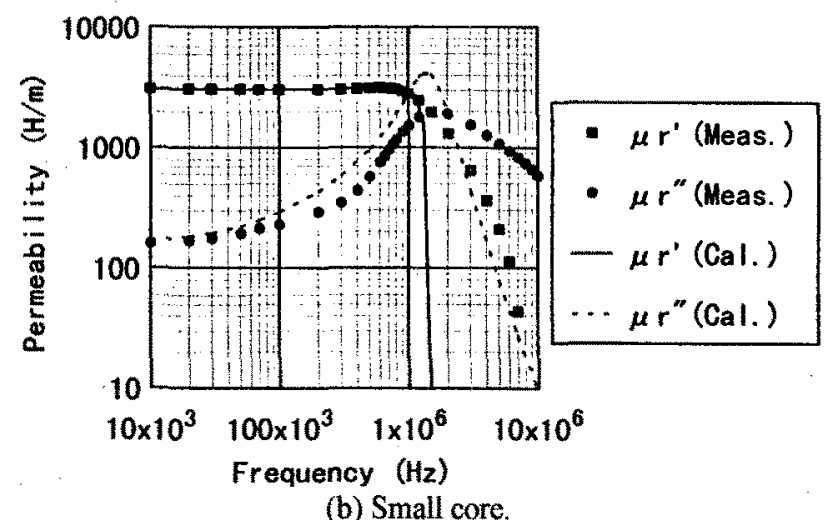

(b) Small core.

Fig. 5. Permeability with taking account of $C_{S}$ by (3).

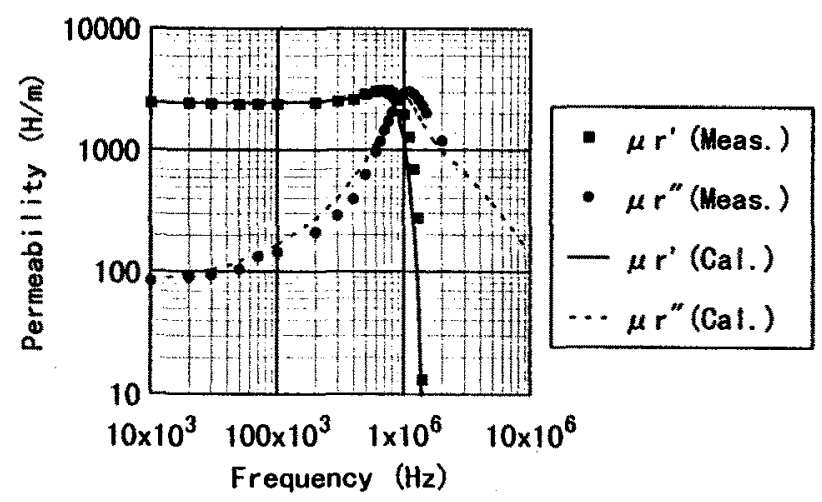

(a) Large core.

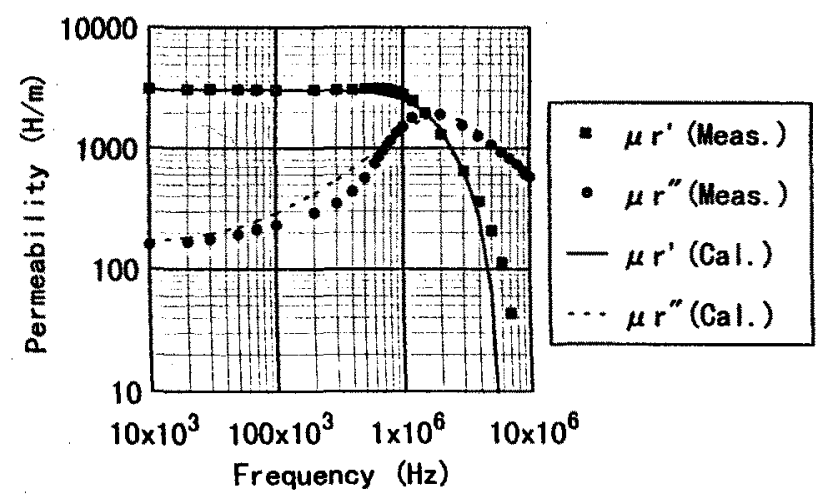

(b) Small core.

Fig. 6, Permeability with taking account of $C_{S}$ by (4).
Assuming $\sigma^{\prime \prime}=\left(65000 \varepsilon_{0}\right) \omega$ (Fitting $\varepsilon_{S}$ has also been carried out and $\varepsilon_{0}$ is the permittivity of free space.) and ignoring $\delta^{\prime \prime}$, the frequency characteristics of the complex permeability of the large core are calculated and plotted in Fig.6(a) with the measured data. With the same condition, the complex permeability for the small core is calculated and plotted in Fig.6(b) with its measured data. In this case, the computational and measured data almost coincide for the large and small cores. This result implies that we find out a consistent and constant value of $\varepsilon_{S}$, in core dimensions, corresponding to that of (4).

\section{CONCLUSIONS}

As for the ferrite we used in this paper, it is found that the frequency characteristics of the complex permeability can be explained by the following factors:

1) Inductance.

2) Resistance corresponding to the magnetic power consumption.

3) Resistance corresponding to the eddy current loss.

4) Capacitance-resistance series element. The resistance corresponds to the power consumption caused by the lead phase current with respect to the voltage.

5) Capacitance where completely (90 degree) lead displacement current, with respect to the exciting voltage, flows. This displacement current is supposed to flow in only grain boundaries.

\section{REFERENCES}

[1] H.Saotome and Y.Sakaki, Trans. IEE of Japan, vol.117-A[8], pp.813-817, 1997.

[2] H.Saotome, T.Kawai, and Y.Sakaki, Trans. IEE of Japan, vol.116-A[6], pp.559-565, 1996.

[3] H.Saotome, and Y.Sakaki, IEEE Trans. Magn., vol.33[1], pp.728-734, 1997.

[4] T.Kawai, H.Saotome, and Y.Sakaki, J. Magn. Soc. Japan, vol.21[4-1], pp.193-198, 1997. 